\title{
Aggressive Lentigo Maligna Successfully Treated with Imiquimod 5\%: A Case Report
}

\author{
Holm, $\mathbf{J}^{1}$, Lorentzen, $\mathbf{H}^{2}$, Stolle, $\mathbf{L}^{1}$ \\ ${ }^{1}$ Department of Plastic Surgery, Vejle Hospital, Odense University Hospital, Denmark \\ ${ }^{2}$ Department of Dermatology, Aarhus University Hospital, Denmark
}

"Corresponding author: Jens Holm, Department of Plastic Surgery, Vejle Hospital, Odense University Hospital, Denmark, E-mail: jensholm87@gmail.com

Citation: Holm, L., et al. Aggressive Lentigo Maligna successfully treated with Imiquimod 5\%: A Case Report. (2016) Invest Dermatol Venereol Res 2(1): 64-66.

Received date: February 19, 2016

Accepted date: April 27, 2016

DOI: $10.15436 / 2381-0858.16 .017$

Published date: May 04, 2016

\begin{abstract}
The use of the topical Imiquimod (IMQ) 5\% cream as a non-surgical alternative to melanoma in situ (MIS) and Lentigo Maligna (LM) is still controversial. Several case reports on this subject are available, but no general consensus exists regarding the efficacy of IMQ for the treatment of MIS.

Here we present a case of a patient successfully treated with topical IMQ 5\% for persistent and aggressive LM despite repetitive surgery. In conclusion, IMQ appears to be a safe alternative to surgery in treating MIS; however, more studies are needed to more precisely determine its role in this specific application.
\end{abstract}

\section{Introduction}

Melanoma in situ (MIS) often occurs in sun-damaged areas of the skin, i.e. head and neck ${ }^{[1]}$. It is a non-invasive lesion confined to the epidermis, accounting for approximately $25 \%$ of all melanomas. Untreated, MIS can progress to invasive melanoma, with an estimated risk approaching $2.2-4.7 \%{ }^{[2]}$.

In Denmark, the standard treatment of MIS and LM is surgical excision with a margin of $5 \mathrm{~mm}$, though LM frequently displays additional subclinical extensions. Radical surgical excision is an option, but not in all cases, as comorbidity, size and/or location of the lesion may challenge the surgeon with a complicated reconstruction - and the patient with a poor functional and cosmetic outcome. Moreover, certain cases of LM may display subclinical margins, and are therefore less likely to be radically excised compared to non-LM MIS. Hence the recurrence rate is $8-20 \%$ after standard surgery, thus questioning the recommended 5 mm excision margin ${ }^{[3]}$.

Therefore, radiotherapy, cryotherapy and Imiquimod have been suggested as non-surgical alternatives ${ }^{[1]}$. IMQ is a potent stimulator of the innate immune system by activating natural killer cells, interferon- $\alpha$, interleukins and tumor necrosis factor- $\alpha^{[4]}$. It was originally used in the treatment of actinic keratosis, superficial basal cell carcinomas and genital warts. IMQ was used in 264 patients with MIS or LN and $220(82 \%)$ of the patients had clinical - and histologic clearance of their lesion according to Ellis et $a l^{[1]}$.

In this case report we present a patient who was successfully cleared of a large and aggressive LM with topically applied $5 \%$ IMQ cream. 


\section{Case Report}

A 91-year-old Caucasian female patient was treated originally treated in 1998 with cryotherapy for a large LM located on her right cheek and lower eyelid measuring approximately seven times four cm. In 2012 and twice in 2014 parts of the patient's in situ lesion transformed clinically in color and shape. Histology confirmed in 2012 that the suspected area of the LM had developed into an invasive Lentigo Malignant Melanoma (LMM) of $0.65 \mathrm{~mm}$ thickness, with no ulceration and Clark's level III. The lesion was excised with $5 \mathrm{~mm}$ margins.

In April 2014 a LMM of 3.06 mm thickness, Clark's level IV, with ulceration and $>=1$ mitosis per mm ${ }^{2}$ was excised from another part of the LM. Several months later yet another LMM of $0.33 \mathrm{~mm}$ thickness and Clark's level II was excised. Re-excision was done according to the Danish Melanoma Guidelines ${ }^{[5]}$, but in all cases, residual in situ lesions were found in the resection margins. Thus, the 91-year-old patient was not interested in further extensive surgery for her aggressive LM. Figure 1 - 4

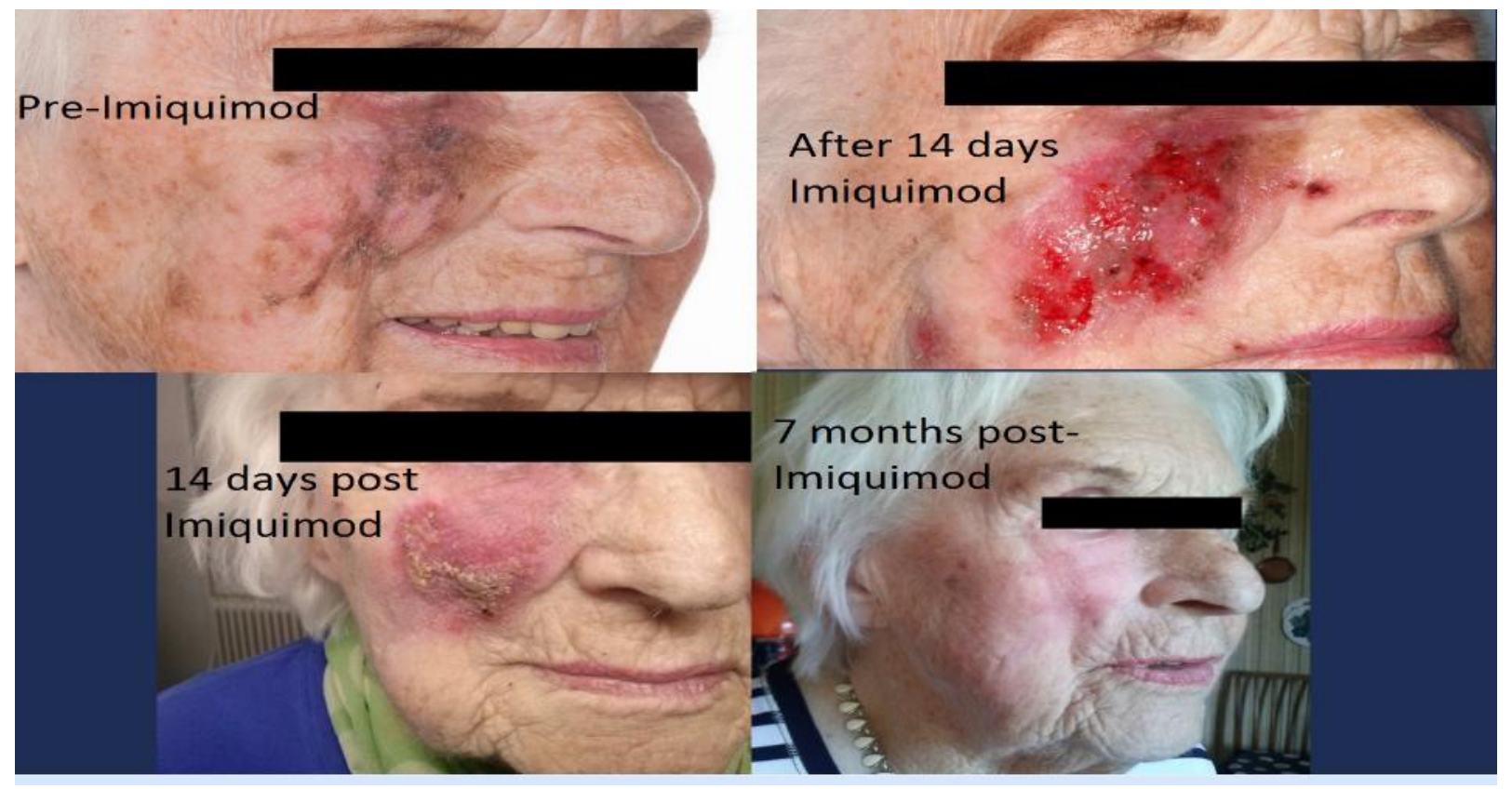

The patient was offered topical IMQ 5\% once a day, five days a week for 12-16 weeks depending on clinical response and tolerance. Within a month of treatment, a yellow crust formed on the lesion along with clear clinical signs of response to the treatment. The treatment continued for more than 20 weeks, and during the last 4 weeks was reduced to 3 times a week due to a minor superficial infection in the lesion along with influenza-like symptoms.

Five months after IMQ, $4 \mathrm{~mm}$ punch biopsies from the 3 invasive LMM areas of the former LM lesion showed complete response based on the presence of inflammatory cells in the biopsies and no sign of malignancy.

Finally, 14 months after the treatment, the latest clinical examination revealed no signs of residual tumor. The patient continues follow-up examinations every 6 months. Figure 5

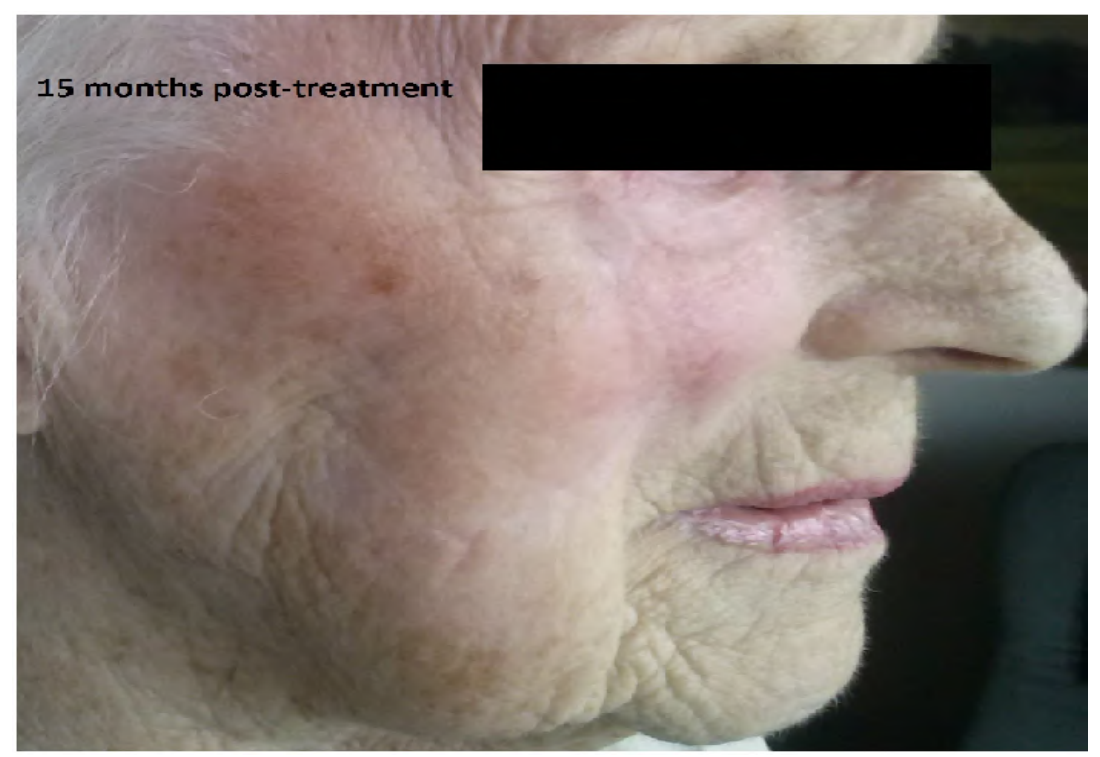




\section{Discussion}

IMQ is generally a well-tolerated treatment. Typical side effects of topically applied IMQ include local erythema, pain, influenza-like symptoms and nausea, though more severe side effects like chronic neuropathic pain, renal failure and Steven Johnson syndrome have been reported in rare cases ${ }^{[4]}$. The side effects typically disappear after a few days without IMQ treatment.

No international accepted treatment protocol for IMQ on MIS is available. Several reports of off-label use of IMQ in the treatment of LM and MIS are available in the literature. Thus, Pandit et al reported a 95\% success rate for 21 of 22 patients treated for MIS positive margins after excision. Ellis et al showed an $82 \%$ success rate. The general consensus is that IMQ is an effective treatment of MIS and LM when radical surgery is not an option, or as either pre- or post-surgery adjuvant treatment. An advantage with IMQ treatment is that it does not rule out other treatment options as other treatments often do. Besides, IMQ leaves the patient with a satisfying cosmetic outcome and no irreversible skin alterations.

Our patient showed clear signs of clinical response to the treatment in form of a severe inflammatory reaction. Inflammation of the treated area appears to be an important predictor of the outcome of the treatment, as several studies report insufficient response in patients without inflammation of the treated area ${ }^{[6]}$.

As more malignant and especially pre-malignant lesions like LM and MIS must be expected in the future ${ }^{[7]}$ due to more extensive sun bathing of the young generation, additional treatment options must be considered. LM and other pre-malignant lesions are often located in the upper part of the face including the eyelids, ears, scalp and neck, and can be both surgically difficult to remove radically and can also leave the patient with a poor cosmetic and functional outcome. Our case showed that IMQ can be efficient in the treatment of MIS/LM, when combining surgery and topical treatment in the management of a complex disease.

\section{Conclusion}

This case report supports the topical use of IMQ as a non-surgical alternative when surgical margins are positive of MIS, and re-excision is not an option. More studies are needed, though, to more precisely define the role of IMQ in the treatment of MIS and LM, i.e. large controlled randomized studies.

\section{Conflict Of Interest: None.}

\section{References}

1. Ellis, L.Z., Cohen, J.L., High, W., et al. Melanoma in situ treated successfully using imiquimod after nonclearance with surgery: review of the literature. (2012) Dermatol Surg 38(6): 937-946.

2. Weinstock, M.A., Sober A.J. The risk of progression of lentigo maligna to lentigo malignant melanoma. (1987) Br J Dermatol 116(3): 303-310.

3. Robinson, J.K. Margin Control for Lentigo Maligna. (1994) J Am Acad Dermatol 31(1): 79-85.

4. Pandit, A.S., Geiger, E.J., Ariyan, S., et al. Using topical imiquimod for the management of positive in situ margins after melanoma resection. (2015) Cancer Med 4(4): 507-512.

5. DMG Guidelines (2013)

6. Van Meurs, T., Van Doorn, R., Kritschig, G., et al. Treatment of lentigo maligna with imiquimod cream: a long-term follow-up study of 10 patients. (2010) Dermatol Surg 36(6): 853-858.

7. Helvind, N.M., Holmich, L.R., Smith, S., et al. Incidence of in situ and invasive Melanoma in Denmark from 1985 Through 2012: A National Database Study of 24,059 Melanoma Cases. (2015) JAMA Dermatol 151(10): 1087-1095.

Online ISSN: $2381-0858$

Journal Title: Investigative Dermatology and Venereology Research Journal Short Name: Invest Dermatol Venereol Res
Ommega Online Publishers

E-mail: editor.dermatology@ommegaonline.org

Website: www.ommegaonline.org 